\title{
Direct linkage of mitochondrial genome variation to risk factors for type 2 diabetes in conplastic strains
}

\author{
Michal Pravenec, ${ }^{1}$ Masaya Hyakukoku, ${ }^{2,3}$ Josef Houstek, ${ }^{1}$ Vaclav Zidek, ${ }^{1}$ \\ Vladimir Landa, ${ }^{1}$ Petr Mlejnek, ${ }^{1}$ Ivan Miksik, ${ }^{1}$ Kristyna Dudová-Mothejzikova, ${ }^{1}$ \\ Petr Pecina, ${ }^{1}$ Marek Vrbacký, ${ }^{1}$ Zdenek Drahota, ${ }^{1}$ Alena Vojtiskova, ${ }^{1}$ Tomas Mracek, ${ }^{1}$ \\ Ludmila Kazdova, ${ }^{4}$ Olena Oliyarnyk, ${ }^{4}$ Jiaming Wang, ${ }^{3}$ Christopher Ho, ${ }^{3}$ Nathan Qi, ${ }^{5}$ \\ Ken Sugimoto, ${ }^{6}$ and Theodore Kurtz ${ }^{3,7}$

\begin{abstract}
${ }^{1}$ Institute of Physiology, Academy of Sciences of the Czech Republic, Prague 142 20, Czech Republic; ${ }^{2}$ Second Department of Medicine, Sapporo Medical University, Sapporo 060-8543, Japan; ${ }^{3}$ Department of Laboratory Medicine, University of California, San Francisco, California 94107, USA; ${ }^{4}$ Institute for Clinical and Experimental Medicine, Prague 140 21, Czech Republic; ${ }^{5}$ Department of Medicine, University of Michigan Medical School, Ann Arbor, Michigan 48109, USA; ${ }^{6}$ Department of Geriatric Medicine, Osaka University Graduate School of Medicine, Osaka 565-0871, Japan
\end{abstract}

\begin{abstract}
Recently, the relationship of mitochondrial DNA (mtDNA) variants to metabolic risk factors for diabetes and other common diseases has begun to attract increasing attention. However, progress in this area has been limited because (1) the phenotypic effects of variation in the mitochondrial genome are difficult to isolate owing to confounding variation in the nuclear genome, imprinting phenomena, and environmental factors; and (2) few animal models have been available for directly investigating the effects of mtDNA variants on complex metabolic phenotypes in vivo. Substitution of different mitochondrial genomes on the same nuclear genetic background in conplastic strains provides a way to unambiguously isolate effects of the mitochondrial genome on complex traits. Here we show that conplastic strains of rats with identical nuclear genomes but divergent mitochondrial genomes that encode amino acid differences in proteins of oxidative phosphorylation exhibit differences in major metabolic risk factors for type 2 diabetes. These results (1) provide the first direct evidence linking naturally occurring variation in the mitochondrial genome, independent of variation in the nuclear genome and other confounding factors, to inherited variation in known risk factors for type 2 diabetes; and (2) establish that spontaneous variation in the mitochondrial genome per se can promote systemic metabolic disturbances relevant to the pathogenesis of common diseases.
\end{abstract}

[Supplemental material is available online at www.genome.org.]

Recently, the potential role of the mitochondrial genome in the pathogenesis of type 2 diabetes and other common diseases has begun to attract increasing attention (Wallace 2005). Mitochondrial DNA is exclusively of maternal origin, and some studies have indicated that the inheritance of type 2 diabetes may be biased toward the maternal lineage (Alcolado et al. 2002). Sun et al. (2003) have estimated that mitochondrial DNA mutations might be involved in $>20 \%$ of cases of type 2 diabetes. In addition, Wilson et al. (2004) have identified a homoplasmic mitochondrial DNA variant associated with the maternal transmission of hypercholesterolemia, hypomagnesemia, and hypertension through four generations of a large, carefully analyzed kindred. Rare forms of maternally transmitted diabetes associated with heteroplasmic mitochondrial DNA variants have also been reported (Mathews and Berdanier 1998; Maassen et al. 2005). These observations provide indirect evidence that sequence variation in the mitochondrial genome might contribute to inherited variation in the risk for type 2 diabetes and related metabolic disorders.

\section{${ }^{7}$ Corresponding author.}

E-mail KurtzT@Labmed2.ucsf.edu; fax (801) 912-3103.

Article published online before print. Article and publication date are at http:// www.genome.org/cgi/doi/10.1101/gr.6548207.
Notwithstanding recent advances in mitochondrial genome research, multiple confounding factors have made it difficult to unequivocally establish a role for mitochondrial DNA variation in the regulation of risk factors for diabetes or other complex metabolic phenotypes. For example, effects of maternal environment or imprinting may contribute to matrilineal transmission patterns of the phenotypes of interest. Variation in copy number and tissue distribution of heteroplasmic mitochondrial DNA mutants can have unpredictable phenotypic consequences. Perhaps the most vexing problem is that the phenotypic expression of even homoplasmic mitochondrial DNA variants may be highly variable because of complex interactions with nuclear gene variants or environmental factors that are difficult to control (Fischel-Ghodsian 2000; Carelli et al. 2003). Thus, in many if not most cases, imperfect correlations between mitochondrial genotype and phenotype have been typically ascribed to incomplete penetrance involving phenomena that are usually not elucidated or explored.

In this study, we investigated whether naturally occurring variation in the mitochondrial genome could be directly linked to risk factors for type 2 diabetes in a rat model in which we have eliminated the confounding effects of variation in the nuclear genome, imprinting phenomena, and environmental factors. We 
accomplished this objective by selectively replacing the mitochondrial genome of the spontaneously hypertensive rat (SHR) with the mitochondrial genome of the Brown Norway (BN) rat to create a novel SHR conplastic strain. Because the SHR conplastic strain and the SHR progenitor strain are genetically identical except for differences confined to their mitochondrial genomes, any phenotypic differences between these two strains can be directly attributed to sequence differences in their mitochondrial DNA. Using this approach, we have identified naturally occurring variants in rat mitochondrial genes encoding proteins of oxidative phosphorylation that are directly linked to alterations in mitochondrial enzyme levels and major risk factors for type 2 diabetes.

\section{Results}

\section{Risk factors for diabetes}

We found that fasting glucose and insulin levels were similar between the SHR conplastic strain and the SHR progenitor strain (Fig. 1A,B). However, after oral glucose loading, both the area under the curve (AUC) for glucose levels and the AUC for insulin levels were significantly greater in the SHR conplastic strain, $664 \pm 9 \mathrm{mmol} / \mathrm{L} / 2 \mathrm{~h}$ and $18.8 \pm 1.3$ $\mathrm{nmol} / \mathrm{L} / 2 \mathrm{~h}$, respectively, than in the SHR progenitor strain, $603 \pm 11 \mathrm{mmol} / \mathrm{L} / 2 \mathrm{~h}$ and $13.6 \pm 0.6$ $\mathrm{nmol} / \mathrm{L} / 2 \mathrm{~h}$, respectively (both $P<0.01$; Fig. $1 \mathrm{~A}, \mathrm{~B}$ ). Thus, replacing the SHR mitochondrial genome with the $\mathrm{BN}$ mitochondrial genome promoted impaired glucose tolerance, a major clinical risk factor for diabetes (Nathan et al. 2007). Fasting and nonfasting triglyceride levels tended to be greater in the conplastic strain than in the progenitor strain, but the differences did not achieve statistical significance (data not shown). The reduced glucose tolerance of the SHR conplastic strain was not associated with excess body weight, as the body weight of the SHR conplastic strain $(283 \pm 10 \mathrm{~g})$ was slightly lower than that of the SHR progenitor strain $(305 \pm 10 \mathrm{~g} ; P<0.01)$.

Because reduced skeletal muscle glycogen synthesis is a characteristic feature of pre-diabetic individuals with suboptimal glucose tolerance and of patients with diabetes (Shulman et al. 1990; Petersen et al. 2004), we also measured glycogen content in skeletal muscle of the SHR conplastic and progenitor strains. In the SHR conplastic strain, skeletal muscle glycogen content was significantly decreased compared to that in the SHR progenitor strain (Fig. 1C). We also measured skeletal muscle ATP levels because reduced muscle ATP synthesis is another metabolic characteristic associated with increased risk for diabetes (Petersen et al. 2004). The ATP level in skeletal muscle of the SHR conplastic strain was significantly decreased compared to that in the SHR progenitor strain (Fig. 1D). These results demonstrate that selective replacement of the mitochondrial genome of the SHR with
B

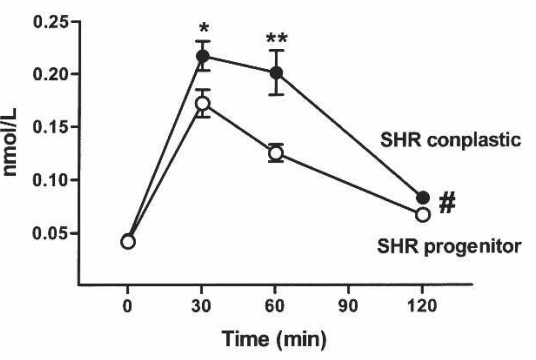

D

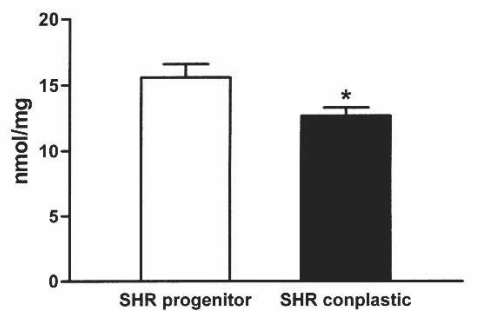

Figure 1. Metabolic differences between the SHR progenitor strain and SHR conplastic strain with identical nuclear genomes but different mitochondrial genomes. $(A)$ Blood glucose levels before and after glucose administration in the SHR progenitor strain (open circles, $n=9$ ) and the SHR conplastic versus $603 \pm 11 \mathrm{mmol} / \mathrm{L} / 2 \mathrm{~h}$, respectively $(P<0.01)$. Significant differences at individual time points significantly greater area under the curve for serum insulin levels than the SHR progenitor strain, in skeletal muscle. Glycogen content in skeletal muscle tissue from the SHR conplastic strain (solid bar $n=12$ ) was significantly lower than in the SHR progenitor strain (open bar, $n=9, P<0.001$ ). (D) ATP content in skeletal muscle. ATP content in skeletal muscle tissue from the SHR conplastic strain (solid bar, $n=6$ ) was lower than in the SHR progenitor strain (open bar, $n=5, P<0.05)$. $\left({ }^{* *}\right) P<0.001 ;\left({ }^{* *}\right)$ $P<0.01 ;\left(^{*}\right) P<0.05$ in individual time-point comparisons between the SHR progenitor and conplastic

the mitochondrial genome of the $\mathrm{BN}$ rat influences several major metabolic risk factors for type 2 diabetes.

In supplemental studies in reciprocally derived female $F_{1}$ hybrids that inherited the BN mitochondrial genome, fasting serum insulin levels $(0.09 \pm 0.02 \mathrm{nmol} / \mathrm{L})$ and triglyceride levels $(1.12 \pm 0.04 \mathrm{mmol} / \mathrm{L})$ were significantly increased compared to those in $F_{1}$ hybrid rats that inherited the SHR mitochondrial genome, $0.05 \pm 0.008 \mathrm{nmol} / \mathrm{L}(P<0.05)$ and $0.89 \pm 0.06$ $\mathrm{mmol} / \mathrm{L}(P<0.01)$, respectively. Despite differences in fasting insulin, the fasting glucose level in rats that inherited the $\mathrm{BN}$ mitochondrial genome $(4.1 \pm 0.2 \mathrm{mmol} / \mathrm{L})$ was not significantly different from that in rats that inherited the SHR mitochondrial genome $(4.5 \pm 0.2 \mathrm{mmol} / \mathrm{L})$. Insulin-stimulated incorporation of glucose into skeletal muscle glycogen in $F_{1}$ rats with the $\mathrm{BN}$ mitochondrial genome was significantly lower than that in $F_{1}$ rats with the SHR mitochondrial genome, $198 \pm 71 \mathrm{nmol}$ glucose/ $\mathrm{g} / 2 \mathrm{~h}$ versus $517 \pm 100 \mathrm{nmol}$ glucose $/ \mathrm{g} / 2 \mathrm{~h}$, respectively $(P<0.05)$. These findings in reciprocally derived female $F_{1}$ hybrids with identical nuclear genomes but different mitochondrial genomes demonstrate maternal transmission bias in several known metabolic risk factors for type 2 diabetes and are consistent with those in the conplastic strain.

\section{Mitochondrial DNA sequence analysis}

Sequence analysis revealed distinct differences in the mitochondrial genomes of the SHR and BN strains. Comparison of the

\section{Genome Research} www.genome.org 
Table 1. Mitochondrial DNA variants encoding amino acid substitutions in SHR and BN strains

\begin{tabular}{|c|c|c|c|c|c|c|}
\hline \multirow[b]{2}{*}{ Gene } & \multirow[b]{2}{*}{ Nucleotide } & \multicolumn{2}{|c|}{ Codon } & \multicolumn{2}{|c|}{ Amino acid } & \multirow[b]{2}{*}{ Number } \\
\hline & & $\mathrm{BN}$ & SHR & $\mathrm{BN}$ & SHR & \\
\hline \multirow[t]{4}{*}{$m t-N d 2$} & 3956 & GTA & GCA & Val & Ala & 18 \\
\hline & 4352 & AAC & AGC & Asn & Ser & 150 \\
\hline & 4633 & ACA & GCA & Thr & Ala & 244 \\
\hline & 4696 & GCA & ACA & Ala & Thr & 265 \\
\hline$m t-\mathrm{Co} 1$ & 5326 & CTC & TTC & Leu & Phe & 2 \\
\hline mt-Atp8 & 7930 & ACC & ATC & Thr & Ile & 58 \\
\hline mt-Atp6 & 8021 & GAA & AAA & Glu & Lys & 35 \\
\hline \multirow[t]{4}{*}{$m t-N d 4$} & 10227 & ATC & ACC & Ile & Thr & 23 \\
\hline & 11225 & GCA & ACA & Ala & Thr & 356 \\
\hline & 11360 & GTC & ATC & Val & Ile & 401 \\
\hline & 11374 & ATC & ATA & Ile & Met & 405 \\
\hline \multirow[t]{2}{*}{$m t-N d 6$} & 13647 & ATT & GTT & Ile & Val & 139 \\
\hline & 13974 & $\mathrm{CTT}$ & TTT & Leu & Phe & 30 \\
\hline \multirow[t]{2}{*}{$m t-C y b$} & 14775 & GAC & AAC & Asp & Asn & 214 \\
\hline & 15135 & ATC & GTC & Ile & Val & 334 \\
\hline
\end{tabular}

complete mitochondrial DNA sequences revealed polymorphisms of potential functional significance in seven of the 13 messenger RNA (mRNA) genes (Table 1), five of the 22 transfer RNA (tRNA) genes (Supplemental Table 1), and both of the ribosomal RNA (rRNA) genes (Supplemental Table 1). None of the observed differences between SHR and BN mtDNA was associated with heteroplasmy. The mRNA variants are predicted to cause amino acid substitutions in mitochondrial cytochrome $c$ oxidase subunit I (COX1), ATP synthase subunit 6 (ATP6), ATP synthase subunit 8 (ATP8), cytochrome $b$ (CYTB), NADH dehydrogenase subunit 2 (ND2), NADH dehydrogenase subunit 4 (ND4), and NADH dehydrogenase subunit 6 (ND6) (Table 1). Some of these substitutions are located within 0-3 amino acid residues of mutation sites known to be associated with human disorders including exercise intolerance and Leber hereditary optic neuropathy (LHON). Of the seven variants affecting five tRNA genes, three are located within $4 \mathrm{bp}$ of human mutation sites associated with mitochondrial encephalopathy and focal segmental glomerulosclerosis. A single variant was detected in the $12 \mathrm{~S}$ rRNA gene $m t-R n 1$, and multiple variants were detected in the 16S rRNA gene $m t-R n 2$ that appear to be distant from sites associated with human clinical disorders. In addition, one variant was identified in the origin of replication, and seven variants were identified in the D-loop region (Supplemental Table 2).

\section{Mitochondrial DNA haplotype analysis}

We performed haplotype analysis by genotyping 43 rat strains including the SHR and BN progenitor strains for a subset of 16 polymorphisms that included variants causing seven amino acid substitutions in six proteins for oxidative phosphorylation, two silent messenger RNA (mRNA) variants, five transfer RNA (tRNA) variants, and two ribosomal RNA (rRNA) variants. This enabled identification of four haplotype groups including two main categories that encompassed 37 out of the 43 strains tested (Table 2). The SHR strain belongs to a minor haplotype group (group 3) containing only four of the strains tested including SHR, WKY, OKA, and GC. The BN haplotype group (group 4) includes only one other strain, DZB/Gro, and this strain is known to have been derived from a cross of $\mathrm{BN}$ rats with $\mathrm{AO}$ rats. All of the SHR variants, synonymous and nonsynonymous, were found in many other strains. However, five of the $\mathrm{BN}$ variants in genes encoding amino acid substitutions in proteins of oxidative phosphorylation were not found in any of the other strains except DZB/Gro known to have been derived from BN rats. These genes included $m t$-Co1, $m t-A t p 8, m t-N d 4, m t-N d 6$, and $m t-C y b$ (Table 2).

We also compared the amino acid substitutions in the SHR and $\mathrm{BN}$ progenitor strains against the corresponding residues in human, guinea pig, mouse, chimpanzee, macaque, cow, dog, opossum, chicken, zebrafish, and stickleback. Several of the variants observed in the SHR versus BN comparison involved residues that are relatively conserved among multiple species. Of particular interest given the enzyme studies described below is that the $\mathrm{BN}$ rat along with the guinea pig and macaque are the only mammals tested that have a leucine at position 2 of COX1 (variant in $m t$-Co1 nucleotide no. 5326) (Tables 1, 2). The SHR and 40 other rat strains as well as the mouse, cow, dog, human, chimpanzee, opossum, and chicken have phenylalanine in this position, whereas zebrafish and stickleback have either threonine or alanine.

\section{Mitochondrial enzyme analysis}

To determine whether the observed sequence variation in the mitochondrial genome was also linked to effects on mitochondrial enzyme activity or alterations in mitochondrial protein levels, we studied isolated mitochondria from the SHR conplastic and progenitor strains. Analysis of enzyme activities of selected respiratory chain complexes revealed significantly lower activity of cytochrome $c$ oxidase (complex IV) in the SHR conplastic strain compared to the SHR progenitor strain

Table 2. Mitochondrial DNA haplotype grouping of 43 strains of rats

\begin{tabular}{lllll}
\hline & \multicolumn{4}{c}{ Genotype $^{\text {a }}$} \\
\cline { 2 - 5 } Nucleotide & Group 1 & Group 2 & Group 3 & Group 4 \\
\hline 1716 & BN & SHR & SHR & BN \\
1918 & SHR & SHR & SHR & BN \\
$4352^{\text {b }}$ & BN & SHR & SHR & BN \\
5200 & BN & SHR & SHR & BN \\
5237 & BN & SHR & SHR & BN \\
5269 & BN & SHR & SHR & BN \\
$5326^{\text {b }}$ & SHR & SHR & SHR & BN \\
$7930^{\text {b }}$ & SHR & SHR & SHR & BN \\
$8021^{\text {b }}$ & BN & SHR & SHR & BN \\
11,194 & BN & SHR & SHR & BN \\
$11,374^{\text {b }}$ & SHR & Wistar & SHR & BN \\
$13,647^{\mathrm{b}}$ & BN & SHR & SHR & BN \\
13,693 & BN & SHR & SHR & BN \\
$13,974^{\mathrm{b}}$ & SHR & SHR & SHR & BN \\
$15,135^{\mathrm{b}}$ & SHR & SHR & SHR & BN \\
15,400 & SHR & SHR & SHR & BN \\
\hline
\end{tabular}

We identified four haplotype groups by genotype analysis of 16 mitochondrial DNA polymorphisms in 43 rat strains including the SHR and BN progenitor strains. The specific variants present at each nucleotide site in the SHR and BN strains are listed in Tables 1 and Supplemental Table 1. aHaplotype Group 1 consisted of 20 strains: $\mathrm{ACl} / \mathrm{Ztm}, \mathrm{BC} / \mathrm{CpbU}, \mathrm{BDE} /$ Han, BDIV/IfZ, BDIX/Han, BH/Ztm, BUF/Han, COP/OlaHsd, DA/Han, F344/Han, NAR/SaU, NEDH/K, PVG/OlaHsdCp, R/A, RHA/Kun, SDL/Ipvc, SPRD-Cu3/Han, SR/Jrlpvc, SS//rlpvc, WF/Han. Group 2 consisted of 17 strains: AS/Ztm, AVN/Orl, BBWB/Mol, BDII/Han, BS/Ztm, LE/Han, LEW/ OlaHsd, LOU/C, MNS/Gro, MW/Hsd, OM/Han, SD/Rij, SDH/Ztu, WF/ Gut, WIST/Nhg, WOK.1A/K, WOK/w/K. Group 3 consisted of four strains: SHR/Ola, OKA/Wsl, GC/Kun, WKY/M. Group 4 consisted of two strains: $\mathrm{BN} / \mathrm{Ola}$, DZB/Gro. The DZB/Gro strain was derived by a cross of $\mathrm{BN}$ rats with $A O$ rats.

${ }^{b}$ Nucleotides causing amino acid substitutions. 
(Fig. 2A). In contrast, we found no strain differences in the activities of succinate:ferricytochrome $c$ oxidoreductase (complex II + III), NADH:ferricytochrome $c$ oxidoreductase, rotenone sensitive (complex I + III), or citrate synthase (Fig. 2A). Consistent with the results in the conplastic strain, we also found that mitochondrial cytochrome $c$ oxidase activity was significantly lower in $F_{1}$ hybrid rats that inherited the BN mitochondrial genome than in $F_{1}$ hybrid rats that inherited the SHR mitochondrial genome, $6484 \pm 127 \mathrm{nmol} / \mathrm{min} / \mathrm{mg}$ protein versus $7137 \pm 46 \mathrm{nmol} / \mathrm{min} / \mathrm{mg}$ protein, respectively $(P<0.0001)$. Thus, the BN strain carries a rare variant in the $m t$-Co1 gene that is not found in $>40$ other strains of laboratory rats (Tables 1,2 ) and that is genetically linked to effects on cytochrome $c$ oxidase activity.

\section{Analysis of mitochondrial proteins and DNA copy number}

Western blot analysis of mitochondrial protein complexes of oxidative phosphorylation showed decreases in both mtDNAencoded (COX2) and nuclear-encoded (COX4) subunits of cytochrome $c$ oxidase (complex IV) in the SHR conplastic strain compared to the SHR progenitor strain (Fig. 2B). The level of COX1 also tended to be lower in the SHR conplastic strain than the SHR progenitor strain, but the difference did not achieve statistical significance. In contrast, none of the proteins tested in the other respiratory chain complexes (complexes I, III, or V) appeared to be reduced in the SHR conplastic strain (Fig. 2B). We did not detect any reductions in mtDNA copy number relative to genomic DNA content or any reductions in tissue citrate synthase activity between the SHR conplastic strain and the SHR progenitor strain (data not shown). These observations indicate that the reductions in cytochrome $c$ oxidase content and enzyme activity linked to the BN mitochondrial genome are relatively selective in nature and not just a consequence of widespread disturbances in mitochondrial biogenesis and/or protein content. Western blot studies of liver mitochondria from reciprocally derived $F_{1}$ hybrid rats also revealed effects of mitochondrial genome variation on the protein content of cytochrome $c$ oxidase. In $F_{1}$ rats that inherited the $\mathrm{BN}$ mitochondrial genome, the mitochondrial content of cytochrome $c$ oxidase subunit 1 was decreased by $25 \%-$ $30 \%$ compared to $F_{1}$ rats that inherited the SHR mitochondrial genome $(P<0.01)$. No significant differences were found between the two groups of $F_{1}$ hybrid rats in the levels of other major respiratory chain proteins tested from liver, heart, or kidney mitochondria.

\section{Discussion}

The potential role of mitochondria dysfunction in the pathogenesis of a variety of common diseases including type 2 diabetes has attracted considerable attention, although cause and effect rela-

\section{A}
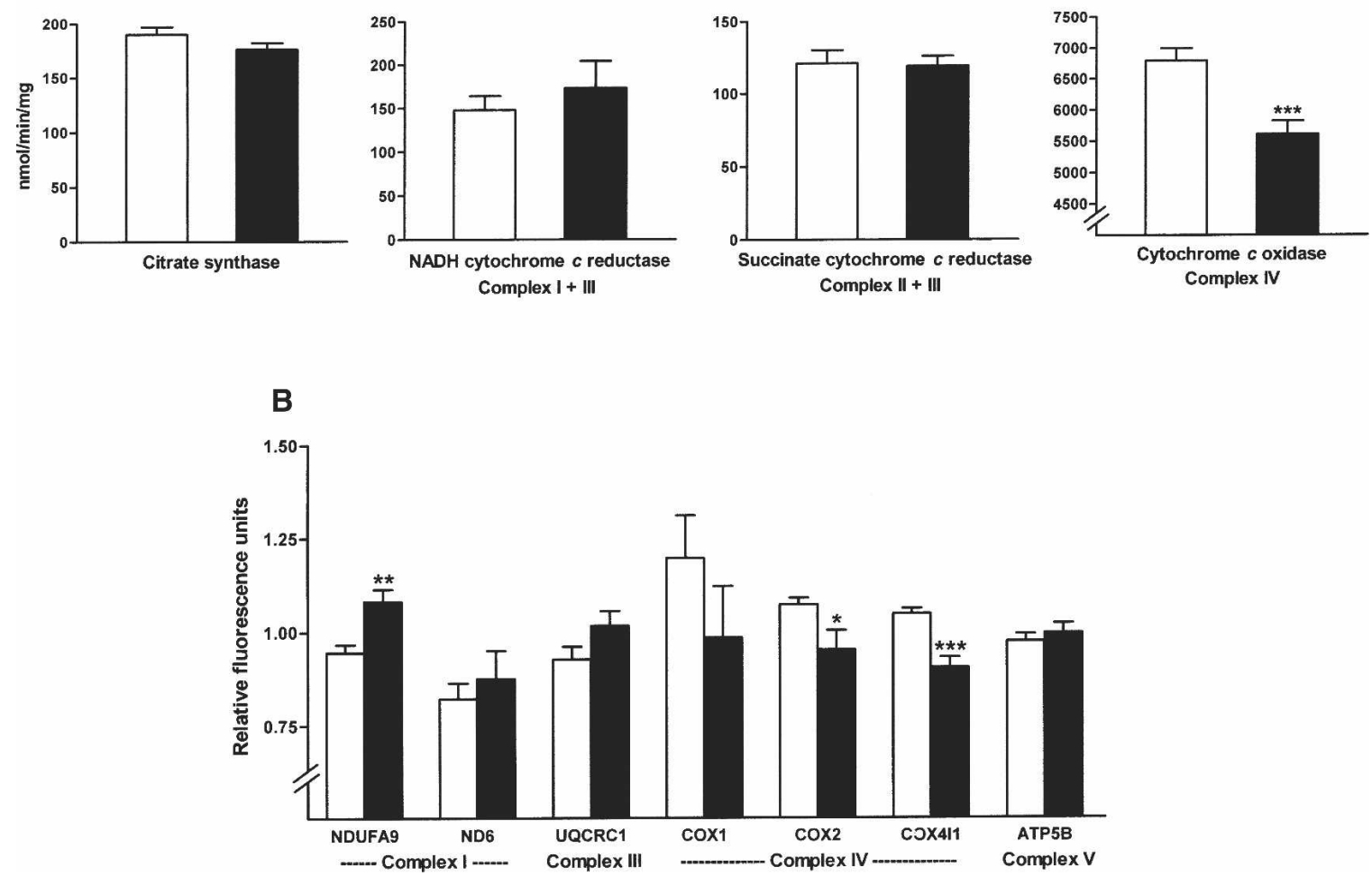

Figure 2. Mitochondrial enzyme activities and protein levels in the SHR progenitor strain and SHR conplastic strain. ( $A$ ) Activity levels of citrate synthase, NADH cytochrome $c$ reductase (NCCR), succinate cytochrome $c$ reductase (SCCR), and cytochrome $c$ oxidase in liver mitochondria isolated from the SHR progenitor strain (open bars) and SHR conplastic strain (solid bars). The activity of cytochrome $c$ oxidase was significantly lower in the SHR conplastic strain $(n=6)$ than the SHR progenitor strain $(n=6, P<0.001)$. (B) Mitochondrial protein content in the SHR progenitor strain $(n=6)$ and SHR conplastic strain $(n=6)$. Results are expressed as relative fluorescence units determined after loading equivalent amounts of total mitochondrial protein for each sample. (NDUFA9) 39-kDa subunit of NADH:ubiquinone oxidoreductase; (ND6) mitochondrial NADH dehydrogenase subunit 6; (UQCRC1) core 1 subunit of ubiquinol:ferricytochrome $c$ oxidoreductase; (COX1) mitochondrial cytochrome $c$ oxidase subunit 1; (COX2) mitochondrial cytochrome $c$ oxidase subunit $2 ;(C O X 4 I 1)$ cytochrome $c$ oxidase subunit 4 isoform $1 ;\left(\right.$ ATP5B) $\beta$-subunit of the $F_{1} F_{0}$ ATP synthase; $(* * *) P<0.001$; (**) $P<0.01 ;\left(^{*}\right) P=0.05$ in comparisons between the SHR progenitor and conplastic strains by Student's $t$-test.

\section{Genome Research}

www.genome.org 
tionships have been difficult to sort out (Petersen et al. 2004; Lowell and Shulman 2005; Wallace 2005; Asmann et al. 2006; Calvo et al. 2006). Owing to variation in the nuclear genome and other confounding factors, it has also been difficult to definitively isolate the effects of naturally occurring variation in the mitochondrial genome alone on risk factors for complex diseases including type 2 diabetes. This may explain why associations between mtDNA variants and risk for type 2 diabetes have been identified in some populations but not others (Saxena et al. 2006; Fuku et al. 2007). Even within relatively homogeneous populations, unrecognized variation in confounding factors could either mimic or impair the ability to detect effects of mtDNA on complex phenotypes. Rare forms of diabetes and other clinical disorders have been associated with inheritance of specific mitochondrial DNA variants; however, the clinical expression of such variants has proven highly variable owing to putative effects of heteroplasmy or interactions with nuclear gene variants or environmental factors that have not been well elucidated or explored (Fischel-Ghodsian 2000; Carelli et al. 2003). Thus, uncertainty has persisted regarding the true impact of naturally occurring variation in the mitochondrial genome on complex metabolic phenotypes in vivo.

In the present studies, we have found that conplastic strains of rats with virtually identical nuclear genomes but divergent mitochondrial genomes exhibit differences in mitochondrial cytochrome $c$ oxidase activity and major metabolic risk factors for type 2 diabetes. Consistent with the results in the conplastic strains, supplemental studies in reciprocally derived female $F_{1}$ hybrids with identical nuclear genomes but different mitochondrial genomes also exhibited differences in cytochrome $c$ oxidase activity and metabolic risk factors for type 2 diabetes. Taken together, the present findings provide direct in vivo evidence that isolated sequence variation in the mitochondrial genome, in the absence of confounding variation in the nuclear genome, physical environment, or imprinting phenomena, can be linked to selective effects on mitochondrial enzyme activity and complex metabolic phenotypes related to risk for diabetes. Moreover, any strain differences in maternal environment that might conceivably have influenced the metabolic and mitochondrial phenotypes in the conplastic rats must also be attributable to strain differences in the mitochondrial genome.

Heretofore, few if any animal models have been available for effectively studying the impact of mtDNA variation on glucose metabolism. For example, in the Goto Kakizaki-Wistar rat model of type 2 diabetes, studies in reciprocal $F_{1}$ populations with identical nuclear genomes but different mitochondrial genomes failed to show any consistent effects on glucose metabolism that might be attributed to sequence variants in the mitochondrial genome (Gill-Randall et al. 2004). In a study of reciprocal $F_{1}$ populations derived by crossing a glucose-intolerant strain of BHE/Cdb rats with Sprague-Dawley rats, Mathews et al. (1999) reported that impaired glucose tolerance was associated with inheritance of a point mutation in the mitochondrial gene encoding ATP synthase subunit 6. However, the Sprague-Dawley rats used to establish the $F_{1}$ populations were not clearly inbred, and the phenotyping studies also included $F_{1}$ males with different Y-chromosomes. In previous studies in congenic strains, we have found that genes on the Y-chromosome can affect metabolic traits in rats (Kren et al. 2001). Therefore, the differences in glucose metabolism reported between $F_{1}$ populations derived from reciprocal crosses of $\mathrm{BHE} / \mathrm{Cdb}$ rats and Sprague-Dawley rats could have been related to variation in the nuclear genome rather than variation in the mitochondrial genome.

Because the mitochondrial genome is inherited as a single unit, the present results do not allow us to directly isolate the potential metabolic effects of the individual DNA variants detected in the sequence analysis of the SHR and BN mitochondrial genomes. However, based on the results of mitochondrial haplotype analysis of 43 rat strains together with the proteomic and enzymatic analyses of respiratory chain complexes in mitochondria isolated from the SHR progenitor and conplastic strains, the present findings strongly suggest a role for variant $m t$-Co1 in the metabolic perturbations linked to the mitochondrial genome. The SHR conplastic rats that harbor a unique variant encoding an amino acid substitution in the COX1 subunit exhibited lower mitochondrial cytochrome $c$ oxidase protein levels and lower mitochondrial cytochrome $c$ oxidase enzyme activity than the SHR progenitor rats that harbor the common variant of $m t$-Co1. In contrast, we did not detect reductions in the mitochondrial content or activity of other respiratory chain proteins in the SHR conplastic strain. We also did not detect any reductions in mtDNA copy number or in citrate synthase activity in the SHR conplastic strain. Thus, the conplastic strain was characterized by relatively selective reductions in mitochondrial cytochrome $c$ oxidase activity rather than a generalized disturbance in mitochondrial biogenesis and or mitochondrial protein content.

The present findings in the SHR progenitor and SHR conplastic strain have established that naturally occurring variation in the mitochondrial genome, in the absence of variation in the nuclear genome and other confounding factors, can influence risk factors for type 2 diabetes similar to those reported in prediabetic subjects with insulin resistance and patients with type 2 diabetes (Shulman et al. 1990; Petersen et al. 2004; Asmann et al. 2006). Thus, these results directly demonstrate that spontaneous variation in the mitochondrial genome per se can promote systemic metabolic disturbances relevant to the pathogenesis of common diseases. The availability of these kinds of conplastic animal models with different mitochondrial genomes on a fixed nuclear genetic background will now permit the derivation of conplastic cell lines in which the mitochondrial DNA haplotypes linked to metabolic effects in vivo can be directly investigated at the cellular level on the same fixed genetic background studied in vivo. These models will also open the door for future studies in which the phenotypic effects of mitochondrial genome variation can be investigated on precisely manipulated nuclear genetic backgrounds in congenic and co-isogenic strains. In this fashion, it should soon be possible to systematically isolate and characterize in vivo functional interactions between inherited variation in the mitochondrial genome and inherited variation in the nuclear genome.

\section{Methods}

\section{Strain derivation}

We selectively replaced the mitochondrial genome of a highly inbred strain of spontaneously hypertensive rats (SHR; SHR/ OlaIpcv strain) with the mitochondrial genome of a highly inbred strain of Brown Norway (BN) rats (BN/Crl strain) to create a novel conplastic strain harboring the $\mathrm{BN}$ mitochondrial genome on the SHR nuclear genetic background. The official name designation of this conplastic strain is SHR/OlaIpcv-mt ${ }^{\mathrm{BN} / \mathrm{Crl}}$ and is referred to hereafter as the SHR conplastic strain. We chose the 
SHR-BN model because mtDNA sequence analysis revealed clear differences in the SHR and BN mitochondrial genomes including homoplasmic variants that encode amino acid substitutions in seven structural proteins involved in oxidative phosphorylation (Tables 1, 2; Supplemental Tables 1, 2). We substituted the BN mitochondrial genome on the background of the SHR because the SHR genetic background has proven favorable for identifying effects of other DNA variants on glucose and lipid metabolism (Pravenec et al. 2001).

We derived the conplastic strain using the "supersonic" breeding strategy of Behringer to accelerate the breeding process (Behringer 1998). In each backcross generation derived from the (male SHR $\times$ female BN) $F_{1}$ hybrids, we superovulated 4 -wk-old females and then mated them overnight with SHR males to generate fertilized ova. We induced superovulation by administration of pregnant mares' serum gonadotropin (PMSG) and human chorionic gonadotropin (hCG) given $48 \mathrm{~h}$ apart. We collected fertilized ova by flushing the oviducts on day 1 of pregnancy, and then we transferred seven to nine two-cell embryos into the oviducts of mature pseudopregnant SHR recipients that had been mated overnight with sterile vasectomized males. This transfer of embryos into the oviducts of pseudopregnant recipients occurred on day 1 of pseudopregnancy and produced pregnant SHR foster mothers that delivered litters $\sim 3$ wk later from which female offspring were obtained for use in creating the next backcross. We superovulated the female offspring when they reached 4 wk of age and immediately mated them to SHR males to repeat the cycle, thereby compressing the length of each backcross generation to a total of $\sim 7 \mathrm{wk}$.

In the present experiments, we studied male conplastic rats that were the product of seven to nine generations of backcrossing. After seven to nine backcross generations, a developing congenic/conplastic line will be identical to its inbred partner across $98.4 \%-99.6 \%$ of the genome (Silver 1995). In the present studies, genotype analysis of polymorphic markers distributed at $~ 10-\mathrm{cM}$ intervals across the nuclear genome confirmed lack of divergence in nuclear DNA between the SHR conplastic strain and the SHR progenitor strain. Thus, there is a very high probability that the conplastic and progenitor strains used in this study are homozygous identical at any given locus in the nuclear genome.

Without sequencing the entire nuclear genomes of both strains, no amount of genotyping can exclude all possible residual heterozygosity with absolute certainty. Therefore, we have also included supplemental studies in female $F_{1}$ hybrid rats derived from reciprocal crosses of the SHR and $\mathrm{BN}$ progenitor strains (see Supplemental Methods describing the studies in female $F_{1}$ hybrids). Female $F_{1}$ offspring derived from reciprocal crosses of highly inbred strains have identical nuclear genomes but different mitochondrial genomes, depending on which maternal strain is used to establish each cross. The $F_{1}$ offspring derived by crossing male SHR $\times$ female $\mathrm{BN}$ rats inherit the $\mathrm{BN}$ mitochondrial genome, whereas the $F_{1}$ offspring derived by crossing male $\mathrm{BN} \times$ female SHR inherit the SHR mitochondrial genome. Because both the SHR and BN progenitor strains have been inbred for well over 40 generations, all $F_{1}$ rats must have identical nuclear genomes. While the studies in female reciprocal $F_{1}$ hybrids can exclude variation in the nuclear genome, they do not exclude potential effects of variation in maternal environment and genomic imprinting phenomena. However, the studies in conplastic strains effectively eliminate the confounding effects of imprinting and maternal environment. Thus, together with the supplemental studies in female reciprocal $F_{1}$ hybrids, the present studies in conplastic rats provide a highly rigorous means of isolating the effects of mitochondrial genome variation on complex phenotypes.

\section{Risk factor phenotyping}

After weaning, we fed the conplastic and progenitor rats standard chow and at $10 \mathrm{wk}$ of age, switched them to a semisynthetic diet (Hope Farms, Netherlands) containing 60\% fructose, as highfructose diets have been shown to increase susceptibility of SHR to genetic variants that influence glucose metabolism (Pravenec et al. 1999). We performed glucose tolerance testing by administering an oral glucose load of $300 \mathrm{mg} / 100 \mathrm{~g}$ body weight after an overnight fast in 12-wk-old male rats. We drew blood from the tail without anesthesia for measurements of glucose, insulin, and triglycerides as previously described (Pravenec et al. 2003). We obtained samples of gastrocnemius muscle by snap-freezing in liquid nitrogen and measured glycogen content enzymatically as glucose using the glucose oxidase method in chloroformmethanol sediment after acid hydrolysis as previously described (Vrana et al. 1978). We also processed snap-frozen samples of gastrocnemius muscle in liquid nitrogen for measurement of ATP content by high-pressure liquid chromatography as previously described (Flachs et al. 2002). The protocols used to study the reciprocal $F_{1}$ hybrids are described in the Supplemental Methods. All experiments were performed in agreement with the Animal Protection Law of the Czech Republic (311/1997), which corresponds fully to the European Community Council recommendations for the use of laboratory animals 86/609/ECC. The experiments were approved by the Ethics Committee of the Institute of Physiology, Czech Academy of Sciences, Prague.

\section{Mitochondrial enzyme activities, protein content, and DNA copy number}

We used spectrophotometric methods (Jesina et al. 2004) to measure enzyme activities of citrate synthase (CS), ferrocytochrome $c$ :oxidoreductase (cytochrome $c$ oxidase [COX], complex IV), succinate:ferricytochrome $c$ oxidoreductase (succinate cytochrome $c$ reductase [SCCR], complexes II + III), and NADH:ferricytochrome $c$ oxidoreductase, rotenone sensitive (NADH cytochrome $c$ reductase [NCCR], complexes I + III) in liver mitochondria isolated using differential centrifugation techniques (Bustamante et al. 1977). Enzyme activity results are expressed per milligram of mitochondrial protein.

We used SDS-PAGE and Western blotting with subunitspecific primary mouse monoclonal antibodies (MitoSciences) to measure mitochondrial levels of a panel of respiratory chain proteins using methods similar to those previously described (Jesina et al. 2004). We measured mitochondrial levels of the $\beta$-subunit of the $\mathrm{F}_{1} \mathrm{~F}_{\mathrm{O}}$ ATP synthase (ATP5B); mitochondrial cytochrome- $c$ oxidase subunit 1 (COX1); mitochondrial cytochrome $c$ oxidase subunit 2 (COX2); cytochrome $c$ oxidase subunit 4 isoform 1 (COX4I1); core 1 subunit of ubiquinol:ferricytochrome $c$ oxidoreductase (UQCRC1); 39-kDa subunit of NADH:ubiquinone oxidoreductase (NDUFA9); and mitochondrial NADH dehydrogenase subunit 6 (ND6). We quantified the protein levels by detection of infrared fluorescence using anti-mouse Alexa Fluor 680-labeled secondary antibodies and an Odyssey Infrared Imaging System (LI-COR Biotechnology). Results are expressed as relative fluorescence units determined after loading equivalent amounts of total mitochondrial protein for each sample.

We used real-time PCR to measure the DNA copy number of mitochondrial genes $m t-C o 1, m t-N d 4, m t-A t p 8$, and $m t-C y b$ relative to the amount of the nuclear gene cytochrome $c$ oxidase, subunit IV, isoform 1 (Cox4i1) as an internal control. For realtime PCR, total DNA was isolated using standard methods and analyzed by real-time PCR testing using QuantiTect SYBR Green reagents (QIAGEN) on an Opticon continuous fluorescence detector (MJ Research) with an annealing temperature of $58^{\circ} \mathrm{C}$.

\section{Genome Research}

www.genome.org 
Mitochondrial DNA levels were normalized relative to the internal genomic DNA control with relative amounts of mtDNA to genomic DNA determined in triplicate using the preferred method of Muller et al. (2002). The primers used to amplify mitochondrial genes were $m t$-Co1 upstream, 5'-GGAGCAGTATTCGC CATCAT; $m t$-Co1 downstream, 5'-CGGCCGTAAGTGAGATG AAT; $m t$-Cyb upstream, 5'-CATCAGTCACCCACATCTGC; $m t-C y b$ downstream, 5'-TGGATGGAATGGGATTTTGT; $m t$-Nd4 upstream, 5'-CTCACAACACACCCCCTACC; $m t$-Nd4 downstream, 5'-TCCCATAACCCCCTAGCTTT; mt-Atp8 upstream, 5'-TGCCA CAACTAGACACATCCA; $m$ t-Atp 8 downstream, 5'-TGTGGGGG TAATGAAAGAGG. The primers used to amplify the nuclear gene Cox4i1 were upstream, 5'-ACTACCCCTTGCCTGATGTG; and downstream, 5'-ACAGATCTGCGCTCACACAC.

\section{DNA sequence and haplotype analysis of mitochondrial genomes}

We amplified the entire rat mitochondrial genome from DNA extracted from liver tissue of a 12-wk-old male SHR (SHR/NCrl strain) using the polymerase chain reaction (PCR) and 34 overlapping primer pairs. Sequencing was performed on an Applied Biosystems 3730xl DNA Analyzer using the BigDye Terminator v 3.1 Cycle sequencing kit (Applied Biosystems). We then searched for variants by comparing the SHR sequence results to the published mitochondrial genome sequence (accession no. AC000022) of the $\mathrm{BN}$ rat (BN/SsNHsdMCW strain). We confirmed all variants causing amino acid changes by resequencing the specific regions of interest in at least three SHR/NCrl rats (two liver samples and one kidney sample) and one BN rat (liver tissue from BN/Crl strain). We performed haplotype analysis by genotyping 43 rat strains including the SHR and BN progenitor strains for a subset of 16 polymorphisms that included variants causing seven amino acid substitutions in six proteins for oxidative phosphorylation, two silent messenger RNA (mRNA) variants, five transfer RNA (tRNA) variants, and two ribosomal RNA (rRNA) variants (Table 2). Genotyping was performed by PCR-restriction fragment length polymorphism analysis (RFLP) or direct sequencing. We used the EMBOSS tool Align (needle; gap penalty $\mathrm{O}=10, \mathrm{Ex}=0.5$, and matrix $=$ DNAfull) for pairwise global alignment of mtDNA sequences and the Transeq tool with the option "Vertebrate Mitochondrial" code for translation of mtDNA sequence to amino acid sequence (Rice et al. 2000) To compare protein sequences, we visualized alignments with the program SeaView (Galtier et al. 1996).

\section{Statistical analysis}

We used the Student's $t$-test to analyze for differences between group means with statistical significance defined as $P<0.05$. All results are expressed as means \pm standard errors of the means (SEM).

\section{Acknowledgments}

This work was supported by National Institutes of Health grants HL35018, HL56028, and HL63709 to T.W.K.; grant $1 \mathrm{M} 6837805002$ from the Ministry of Education, Youth, and Sports of the Czech Republic; grants 303/07/0781 and 301/06/ 0028 from the Grant Agency of the Czech Republic; grants UK24 and 26/2005 from Charles University; Prague, research project AV0Z 50110509; grant MZ000023001 grant from the Ministry of Health of the Czech Republic; and by the European Commission within the Sixth Framework Programme through the Integrated Project EURATools (contract no. LSHG-CT-2005-019015). M.P. is an international research scholar of the Howard Hughes Medical Institute.

\section{References}

Alcolado, J.C., Laji, K., and Gill-Randall, R. 2002. Maternal transmission of diabetes. Diabet. Med. 19: 89-98.

Asmann, Y.W., Stump, C.S., Short, K.R., Coenen-Schimke, J.M., Guo, Z., Bigelow, M.L., and Nair, K.S. 2006. Skeletal muscle mitochondrial functions, mitochondrial DNA copy numbers, and gene transcript profiles in type 2 diabetic and nondiabetic subjects at equal levels of low or high insulin and euglycemia. Diabetes 55: 3309-3319.

Behringer, R. 1998. Supersonic congenics? Nat. Genet. 18: 108.

Bustamante, E., Soper, J.W., and Pedersen, P.L. 1977. A high-yield preparative method for isolation of rat liver mitochondria. Anal. Biochem. 80: 401-408.

Calvo, S., Jain, M., Xie, X., Sheth, S.A., Chang, B., Goldberger, O.A., Spinazzola, A., Zeviani, M., Carr, S.A., and Mootha, V.K. 2006. Systematic identification of human mitochondrial disease genes through integrative genomics. Nat. Genet. 38: 576-582.

Carelli, V., Giordano, C., and d'Amati, G. 2003. Pathogenic expression of homoplasmic mtDNA mutations needs a complex nuclear-mitochondrial interaction. Trends Genet. 19: 257-262.

Fischel-Ghodsian, N. 2000. Homoplasmic mitochondrial DNA diseases as the paradigm to understand the tissue specificity and variable clinical severity of mitochondrial disorders. Mol. Genet. Metab. 71: 93-99.

Flachs, P., Novotny, J., Baumruk, F., Bardova, K., Bourova, L., Miksik, I., Sponarova, J., Svoboda, P., and Kopecky, J. 2002. Impaired noradrenaline-induced lipolysis in white fat of aP2-Ucp1 transgenic mice is associated with changes in G-protein levels. Biochem. J. 364: $369-376$.

Fuku, N., Park, K.S., Yamada, Y., Nishigaki, Y., Cho, Y.M., Matsuo, H., Segawa, T., Watanabe, S., Kato, K., Yokoi, K., et al. 2007. Mitochondrial haplogroup N9a confers resistance against Type 2 diabetes in Asians. Am. J. Hum. Genet. 80: 407-415.

Galtier, N., Gouy, M., and Gautier, C. 1996. SEAVIEW and PHYLO_WIN: Two graphic tools for sequence alignment and molecular phylogeny. Comput. Appl. Biosci. 12: 543-548.

Gill-Randall, R.J., Adams, D., Ollerton, R.L., and Alcolado, J.C. 2004. Is human Type 2 diabetes maternally inherited? Insights from an animal model. Diabet. Med. 21: 759-762.

Jesina, P., Tesarova, M., Fornuskova, D., Vojtiskova, A., Pecina, P., Kaplanova, V., Hansikova, H., Zeman, J., and Houstek, J. 2004. Diminished synthesis of subunit a (ATP6) and altered function of ATP synthase and cytochrome $c$ oxidase due to the mtDNA $2 \mathrm{bp}$ microdeletion of TA at positions 9205 and 9206. Biochem. J. 383: $561-571$.

Kren, V., Qi, N., Krenova, D., Zidek, V., Sladka, M., Mikova, B., Horky, K., Bonne, A., Van Lith, H., Van Zutphen, B., et al. 2001. Y chromosome transfer induces changes in blood pressure and blood lipids in SHR. Hypertension 37: 1147-1152.

Lowell, B.B. and Shulman, G.I. 2005. Mitochondrial dysfunction and type 2 diabetes. Science 307: 384-387.

Maassen, J.A., Janssen, G.M., and Hart, L.M.T. 2005. Molecular mechanisms of mitochondrial diabetes (MIDD). Ann. Med. 37: 213-221.

Mathews, C.E. and Berdanier, C.D. 1998. Noninsulin-dependent diabetes mellitus as a mitochondrial genomic disease. Proc. Soc. Exp. Biol. Med. 219: 97-108.

Mathews, C.E., McGraw, R.A., Dean, R., and Berdanier, C.D. 1999. Inheritance of a mitochondrial DNA defect and impaired glucose tolerance in BHE/Cdb rats. Diabetologia 42: 35-40.

Muller, P.Y., Janovjak, H., Miserez, A.R., and Dobbie, Z. 2002. Processing of gene expression data generated by quantitative real time RT-PCR. Biotechniques 32: 1372-1379. Erratum. 33: 514.

Nathan, D.M., Davidson, M.B., DeFronzo, R.A., Heine, R.J., Henry, R.R., Pratley, R., and Zinman, B. 2007. Impaired fasting glucose and impaired glucose tolerance: Implications for care. Diabetes Care 30: $753-759$.

Petersen, K.F., Dufour, S., Befroy, D., Garcia, R., and Shulman, G.I. 2004 Impaired mitochondrial activity in the insulin-resistant offspring of patients with type 2 diabetes. N. Engl. J. Med. 350: 664-671.

Pravenec, M., Zidek, V., Simakova, M., Kren, V., Krenova, D., Horky, K., Jachymova, M., Mikova, B., Kazdova, L., Aitman, T.J., et al. 1999. Genetics of Cd36 and the clustering of multiple cardiovascular risk factors in spontaneous hypertension. J. Clin. Invest. 103: 1651-1657. Pravenec, M., Landa, V., Zidek, V., Musilova, A., Kren, V., Kazdova, L., Aitman, T.J., Glazier, A.M., Ibrahimi, A., Abumrad, N.A., et al. 2001. 
Pravenec et al.

Transgenic rescue of defective Cd36 ameliorates insulin resistance in spontaneously hypertensive rats. Nat. Genet. 27: 156-158.

Pravenec, M., Kazdova, L., Landa, V., Zidek, V., Mlejnek, P., Jansa, P. Wang, J., Qi, N., and Kurtz, T.W. 2003. Transgenic and recombinant resistin impair skeletal muscle glucose metabolism in the spontaneously hypertensive rat. J. Biol. Chem. 278: 45209-45215.

Rice, P., Longden, I., and Bleasby, A. 2000. EMBOSS: The European Molecular Biology Open Software Suite. Trends Genet. 16: 276-277.

Saxena, R., de Bakker, P.I., Singer, K., Mootha, V., Burtt, N., Hirschhorn, J.N., Gaudet, D., Isomaa, B., Daly, M.J., Groop, L., et al. 2006. Comprehensive association testing of common mitochondrial DNA variation in metabolic disease. Am. J. Hum. Genet. 79: 54-61.

Shulman, G.I., Rothman, D.L., Jue, T., Stein, P., DeFronzo, R.A., and Shulman, R.G. 1990. Quantitation of muscle glycogen synthesis in normal subjects and subjects with non-insulin-dependent diabetes by ${ }^{13} \mathrm{C}$ nuclear magnetic resonance spectroscopy. N. Engl. J. Med. 322: 223-228.

Silver, L. 1995. Mouse genetics: Concepts and applications. Oxford
University Press, Oxford.

Sun, F., Cui, J., Gavras, H., and Schwartz, F. 2003. A novel class of tests for the detection of mitochondrial DNA-mutation involvement in diseases. Am. J. Hum. Genet. 72: 1515-1526.

Vrana, A., Poledne, R., Fabry, P., and Kazdova, L. 1978. Palmitate and glucose oxidation by diaphragm of rats with fructose-induced hypertriglyceridemia. Metabolism 27: 885-888.

Wallace, D.C. 2005. A mitochondrial paradigm of metabolic and degenerative diseases, aging, and cancer: A dawn for evolutionary medicine. Annu. Rev. Genet. 39: 359-407.

Wilson, F.H., Hariri, A., Farhi, A., Zhao, H., Petersen, K.F., Toka, H.R., Nelson-Williams, C., Raja, K.M., Kashgarian, M., Shulman, G.I., et al. 2004. A cluster of metabolic defects caused by mutation in a mitochondrial tRNA. Science 306: 1190-1194.

Received March 29, 2007; accepted in revised form June 27, 2007.

1326 Genome Research

www.genome.org 
Genome Research 17: 1319-1326 (2007)

\section{Direct linkage of mitochondrial genome variation to risk factors for type 2 diabetes in conplastic strains}

Michal Pravenec, Masaya Hyakukoku, Josef Houstek, Vaclav Zidek, Vladimir Landa, Petr Mlejnek, Ivan Miksik, Kristyna Dudová-Mothejzikova, Petr Pecina, Marek Vrbacký, Zdenek Drahota, Alena Vojtiskova, Tomas Mracek, Ludmila Kazdova, Olena Oliyarnyk, Jiaming Wang, Christopher Ho, Nathan Qi, Ken Sugimoto, and Theodore Kurtz

The authors have discovered an error in the presentation of the mitochondrial DNA haplotyping analysis described in Table 2 and wish to correct the data. The row that displays genotypes at nucleotide position 11,374 is incorrect and should be replaced by a new row that displays genotypes at nucleotide position 11,360. The corrected Table 2 is reprinted below. The authors apologize for any inconvenience this error may have caused other investigators in the field.

\begin{tabular}{|c|c|c|c|c|}
\hline \multirow[b]{2}{*}{ Nucleotide } & \multicolumn{4}{|c|}{ Genotype $^{a}$} \\
\hline & Group 1 & Group 2 & Group 3 & Group 4 \\
\hline 1716 & $\mathrm{BN}$ & SHR & SHR & $\mathrm{BN}$ \\
\hline 1918 & SHR & SHR & SHR & BN \\
\hline $4352^{b}$ & $\mathrm{BN}$ & SHR & SHR & $\mathrm{BN}$ \\
\hline 5200 & BN & SHR & SHR & BN \\
\hline 5237 & $\mathrm{BN}$ & SHR & SHR & $\mathrm{BN}$ \\
\hline 5269 & $\mathrm{BN}$ & SHR & SHR & $\mathrm{BN}$ \\
\hline $5326^{b}$ & SHR & SHR & SHR & $\mathrm{BN}$ \\
\hline $7930^{\mathrm{b}}$ & SHR & SHR & SHR & $\mathrm{BN}$ \\
\hline $8021^{b}$ & $\mathrm{BN}$ & SHR & SHR & $\mathrm{BN}$ \\
\hline 11,194 & $\mathrm{BN}$ & SHR & SHR & $\mathrm{BN}$ \\
\hline $11,360^{b}$ & $\mathrm{BN}$ & $\mathrm{BN}$ & SHR & $\mathrm{BN}$ \\
\hline $13,647^{b}$ & $\mathrm{BN}$ & SHR & SHR & BN \\
\hline 13,693 & $\mathrm{BN}$ & SHR & SHR & $\mathrm{BN}$ \\
\hline $13,974^{b}$ & SHR & SHR & SHR & BN \\
\hline $15,135^{b}$ & SHR & SHR & SHR & $\mathrm{BN}$ \\
\hline 15,400 & SHR & SHR & SHR & $\mathrm{BN}$ \\
\hline
\end{tabular}

We identified four haplotype groups by genotype analysis of 16 mitochondrial DNA polymorphisms in 43 rat strains including the SHR and BN progenitor strains. The specific variants present at each nucleotide site in the SHR and BN strains are listed in Tables 1 and Supplemental Table 1. a Haplotype Group 1 consisted of 20 strains: ACl/Ztm, BC/CpbU, BDE/ Han, BDIV/Ifz, BDIX/Han, BH/Ztm, BUF/Han, COP/OlaHsd, DA/Han, F344/Han, NAR/SaU, NEDH/K, PVG/OlaHsdCp, R/A, RHA/Kun, SDL/Ipvc, SPRD-Cu3/Han, SR/Jrlpvc, SS/Jrlpvc, WF/Han. Group 2 consisted of 17 strains: AS/Ztm, AVN/Orl, BBWB/Mol, BDIl/Han, BS/Ztm, LE/Han, LEW/ OlaHsd, LOU/C, MNS/Gro, MW/Hsd, OM/Han, SD/Rij, SDH/Ztu, WF/ Gut, WIST/Nhg, WOK.1A/K, WOK/w/K. Group 3 consisted of four strains: SHR/Ola, OKA/Wsl, GC/Kun, WKY/M. Group 4 consisted of two strains: $\mathrm{BN} / \mathrm{Ola}, \mathrm{DZB} / \mathrm{Gro}$. The $\mathrm{DZB} / \mathrm{Gro}$ strain was derived by a cross of $\mathrm{BN}$ rats with $\mathrm{AO}$ rats.

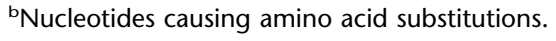


Genome Research 18: 729-741 (2008)

Insights from the complete genome sequence of Mycobacterium marinum on the evolution of Mycobacterium tuberculosis

Timothy P. Stinear, Torsten Seemann, Paul F. Harrison, Grant A. Jenkin, John K. Davies, Paul D.R. Johnson, Zahra Abdellah, Claire Arrowsmith, Tracey Chillingworth, Carol Churcher, Kay Clarke, Ann Cronin, Paul Davis, Ian Goodhead, Nancy Holroyd, Kay Jagels, Angela Lord, Sharon Moule, Karen Mungall, Halina Norbertczak, Michael A. Quail, Ester Rabbinowitsch, Danielle Walker, Brian White, Sally Whitehead, Pamela L.C. Small, Roland Brosch, Lalita Ramakrishnan, Michael A. Fischbach, Julian Parkhill, and Stewart T. Cole

FadB was inadvertently omitted from the sentence on page 731 describing the enzymes involved in betaoxidation in M. tuberculosis. The sentence should have read:

"FadD (acyl-CoA synthetase) catalyses the formation of acyl-CoA thioesters that are then processively oxidized by FadE, EchA, FadB, and FadA (Cole et al. 1998)."

In addition, the page range for the Hagedorn and Soldati 2007 reference was incorrect, the corrected reference is as follows:

Hagedorn, M. and Soldati, T. 2007. Flotillin and RacH modulate the intracellular immunity of Dictyostelium to Mycobacterium marinum infection. Cell Microbiol. 9: 2716-2733.

The authors apologize for any confusion these errors may have caused. 


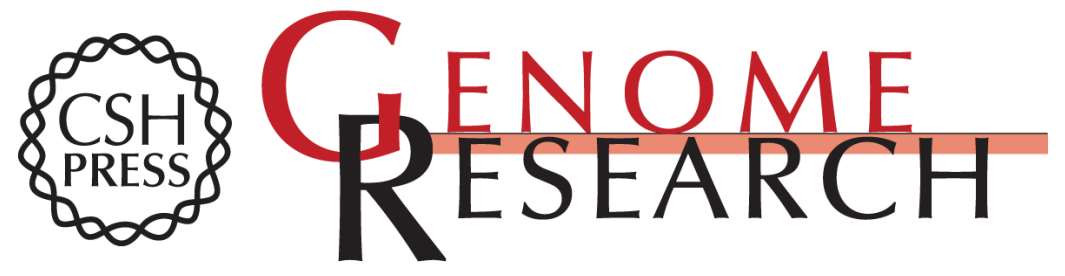

\section{Direct linkage of mitochondrial genome variation to risk factors for type 2 diabetes in conplastic strains}

Michal Pravenec, Masaya Hyakukoku, Josef Houstek, et al.

Genome Res. 2007 17: 1319-1326 originally published online August 10, 2007

Access the most recent version at doi:10.1101/gr.6548207

Supplemental
Material http://genome.cshlp.org/content/suppl/2007/09/05/gr.6548207.DC1

Related Content Errata

Genome Res. October , 2008 18: 1680-1681

References This article cites 30 articles, 6 of which can be accessed free at: http://genome.cshlp.org/content/17/9/1319.full.html\#ref-list-1

Articles cited in:

http://genome.cshlp.org/content/17/9/1319.full.html\#related-urls

\section{License}

Email Alerting Receive free email alerts when new articles cite this article - sign up in the box at the Service top right corner of the article or click here.

\section{Affordable, Accurate Sequencing.}

